\title{
Lasing mode pattern of a quantum cascade photonic crystal surface-emitting microcavity laser
}

\section{Citation}

Srinivasan, Kartik, Oskar Painter, Raffaele Colombelli, Claire Gmachl, Donald M. Tennant, A. Michael Sergent, Deborah L. Sivco, Alfred Y. Cho, Mariano Troccoli, and Federico Capasso. 2004. "Lasing Mode Pattern of a Quantum Cascade Photonic Crystal Surface-Emitting Microcavity Laser." Applied Physics Letters 84 (21): 4164-66. https://doi.org/10.1063/1.1755423.

\section{Permanent link}

http://nrs.harvard.edu/urn-3:HUL.InstRepos:41371589

\section{Terms of Use}

This article was downloaded from Harvard University's DASH repository, and is made available under the terms and conditions applicable to Other Posted Material, as set forth at http:// nrs.harvard.edu/urn-3:HUL.InstRepos:dash.current.terms-of-use\#LAA

\section{Share Your Story}

The Harvard community has made this article openly available. Please share how this access benefits you. Submit a story.

Accessibility 


\title{
Lasing mode pattern of a quantum cascade photonic crystal surface-emitting microcavity laser
}

\author{
Kartik Srinivasan ${ }^{\text {a) }}$ and Oskar Painter \\ Department of Applied Physics, California Institute of Technology, Pasadena, California 91125 \\ Raffaele Colombelli, ${ }^{\text {b) }}$ Claire Gmachl, ${ }^{c}$ Donald M. Tennant, ${ }^{d)}$ A. Michael Sergent, \\ Deborah L. Sivco, and Alfred Y. Cho \\ Bell Laboratories, Lucent Technology, 600 Mountain Avenue, Murray Hill, New Jersey 07974 \\ Mariano Troccoli and Federico Capasso \\ Division of Engineering and Applied Sciences, Harvard University, Cambridge, Massachusetts 02138
}

(Received 26 January 2004; accepted 5 April 2004; published online 6 May 2004)

\begin{abstract}
The identification of the lasing mode within a quantum cascade photonic crystal microcavity laser emitting at $\lambda \sim 8 \mu \mathrm{m}$ is presented. The symmetry of the lasing mode is determined by the position of nodal lines within micro-bolometer camera measurements of its polarized spatial distribution. Full three-dimensional finite-difference time-domain simulations are also performed, and the resulting vertically emitted radiation field pattern is seen to follow the experimental results closely.

(C) 2004 American Institute of Physics. [DOI: 10.1063/1.1755423]
\end{abstract}

In recent work, ${ }^{1}$ planar photonic crystal (PC) microcavities ${ }^{2}$ were embedded in a quantum cascade $(\mathrm{QC})$ heterostructure, creating a QC PC surface-emitting microcavity laser (QC-PCSEL). Although significant progress remains to be made in terms of laser performance, the inherent open cavity architecture, compact form-factor, and electrical injection of these PC microcavity lasers makes them interesting for a diverse set of applications, such as on-chip integration and miniaturization of QC lasers, multiwavelength laser and detector arrays, and microspectroscopy of complex molecules in the mid- to far-infrared. ${ }^{3,4}$ In the QC-PCSEL cavity geometry employed in Ref. 1 [Fig. 1(a)], the PC structure serves both as a means to provide optical feedback and to diffract light vertically from the semiconductor surface. The laser operates on a band-edge state, i.e., a low group velocity mode created in flatband regions of energymomentum space as a result of the distributed feedback effect of the lattice. In this letter, we describe how to identify the lasing band-edge state through a combination of experimental techniques and theoretical analysis. These include far-field intensity measurements, polarized intensity measurements with a micro-bolometer camera, and threedimensional (3D) finite-difference time-domain (FDTD) simulations. This procedure of laser mode identification is based largely upon symmetry arguments, and it could prove a very general and powerful technique for future studies of PC microcavity lasers.

The details of the device design and measurement are given elsewhere (Ref. 1, and references therein). For our purposes here, the key background information is an over-

\footnotetext{
a)Electronic mail: kartik@caltech.edu

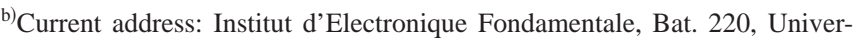
site Paris-Sud, 91405 Orsay, France.

${ }^{c)}$ Current address: Department of Electrical Engineering, Princeton University, Princeton, NJ 08544.

d)Also affiliated with: New Jersey Nanotechnology Consortium, Murray Hill, NJ 07974.
}

view of the principles of the device operation and an understanding of the PC design strategy. Figure 1(a) shows a schematic view of a QC-PCSEL device. Electronic current transport through a cascade of active regions and injectors within the QC heterostructure results in photon emission at $\lambda \sim 8 \mu \mathrm{m}$. Optical feedback is provided by a PC microcavity consisting of an array of air holes that has been etched through the active region and bottom cladding of the QC heterostructure. The lattice of air holes provides distributed Bragg reflection in two dimensions parallel to the chip. For the devices considered in Ref. 1, a bound surface plasmon at the interface between the $\mathrm{QC}$ heterostructure and a thin $\mathrm{Ti} / \mathrm{Au}(10 / 100 \mathrm{~nm})$ metal layer provides the necessary verti-
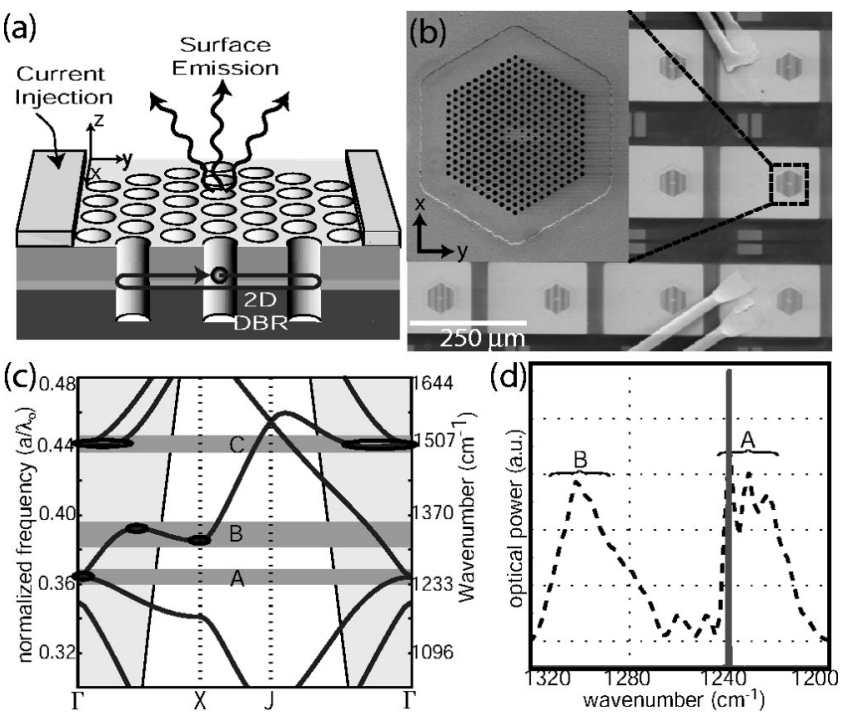

FIG. 1. (a) Schematic of a QC-PCSEL device. (b) Scanning electron microscope image of an array of QC-PCSELs (inset shows a zoomed in top-view of a device). (c) Calculated two-dimensional in-plane TM band structure. The flatband regions $(A, B$, and $C)$ are indicated by dark gray bands. (d) Subthreshold (dashed line) and lasing (solid line) emission spectra from a QC-PCSEL with lattice geometry tailored for emission centered at the $A$ flatband region. 
cal optical confinement. The deep, vertical etch into the bottom cladding layer reduces diffraction losses into the substrate $^{5}$ and provides strong in-plane optical feedback. An array of fully-processed QC-PCSEL devices is shown in Fig. 1(b). The PC lattice consists of a hexagonal array of air holes, chosen primarily due to its connected nature (unlike a lattice of rods, for example), necessary for efficient electrical injection.

The intersubband transitions in quantum wells are naturally TM-polarized (electric field normal to the epitaxial layers). Using the plane-wave expansion method, ${ }^{6}$ we thus calculate the in-plane band structure for TM modes, shown in Fig. 1(c) for a device with hole radius ( $r$ ) to lattice constant (a) ratio $r / a=0.30$, and with an effective index $n_{\text {eff }}=3.35$ taken to account for vertical waveguiding. Highlighted in this band structure are three frequency regions of interest, labeled $A, B$, and $C$, which overlap flatband regions from which band-edge resonant states may be formed. Some of these flatband regions lie within the air light-cone, allowing for light to be vertically radiated into the air. Choosing $a \sim 3$ $\mu \mathrm{m}$ for a hexagonal lattice with $r / a=0.30$ aligns these flatband regions with the QC material gain spectrum $(\lambda \sim 8 \mu \mathrm{m})$. As described in Ref. 1, low-temperature electroluminescence measurements of microfabricated devices reveal three sets of emission peaks, corresponding to regions $A, B$, and $C$ in Fig. 1(c). Figure 1(d) shows the subthreshold and lasing emission spectra for a device with lattice geometry chosen to align the gain peak to the $A$ flatband region. Lasing always originated from the highest frequency (wave number) resonance within the $A$-peak.

The first step in the process of identification of the laser mode is to determine its symmetry. Group theory serves as a powerful tool to categorize the eigenmodes of a given electromagnetic system according to its spatial symmetries. ${ }^{7-9}$ Here, we consider the behavior of the laser mode under reflection about the cavity's $\hat{x}$ and $\hat{y}$ axes [see Fig. 1(b) for the definition of these axes with respect to the cavity]. The polarized spatial distribution of the laser's vertically emitted field intensity is studied by placing a polarizer in front of a micro-bolometer camera fitted with a lens [Figs. 2(a) and 2(b)]. The nodal lines (lines of near-zero intensity in the images) along the $\hat{x}$ and $\hat{y}$ axes of Fig. 2(b) for the $\hat{y}$-polarized intensity pattern are consistent exclusively with an electromagnetic field mode which is odd (parity -1) under a mirror symmetry about the $\hat{y}$ axis and which is even (parity +1$)$ under a mirror symmetry about the $\hat{x}$ axis. In the notation of group theory, ${ }^{7}$ such a mode is said to have $B_{1}$ symmetry, where $B_{1}$ is the label of one of the irreducible representations of the point group of a hexagon or rectangle. ${ }^{10}$ A similar conclusion is reached by studying the $\hat{x}$-polarized intensity pattern of Fig. 2(a), which has antinodes along both the $\hat{x}$ and $\hat{y}$ axis. Thus, the two polarized intensity patterns of Figs. 2(a) and 2(b) indicate that the laser emission is single mode and of $B_{1}$ symmetry.

To better understand the vertical emission characteristics of the PC microcavity modes, full 3D-FDTD simulations were performed. The hole depth was taken to be $5 \mu \mathrm{m}$ and a 200-nm-thick idealized "perfectly conducting" metal top contact was used to guide the TM surface wave (at a wavelength of $8 \mu \mathrm{m}$ this is a reasonable approximation for a gold Downloaded 14 Dec 2005 to 131.215.225.171. Redistribution subject

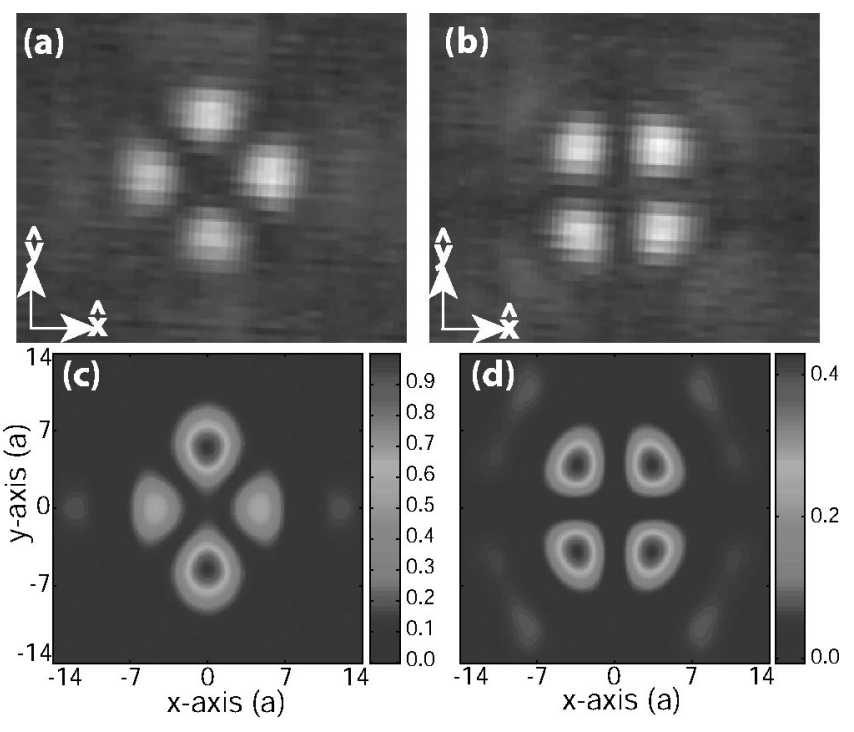

FIG. 2. (a) Polarized emission pattern (taken in a plane close to the nearfield of the PC cavity surface) of a typical lasing device for an electric field polarization along the (a) $\hat{x}$ axis and (b) $\hat{y}$ axis of the cavity. FDTDgenerated (c) $\hat{x}$-polarized, and (d) $\hat{y}$-polarized vertical emission pattern at a few wavelengths above the PC cavity (adapted from Ref. 1).

metal contact $\left.{ }^{11,12}\right)$. To reduce the size and time of the simulation, the cavity was limited to 6 periods of the hexagonal lattice as opposed to the 10 periods used in the experiment. Mirror boundary conditions were used to project the modes of the hexagonally symmetric cavity onto a basis compatible with the symmetry of a rectangle whose principal axes lie along the $\hat{x}$ and $\hat{y}$ axis of the PC microcavity.

The highest frequency resonant mode of the $A$ peak was found to be of $B_{1}$ symmetry (we refer to this mode simply as the $B_{1}$ mode from here on). This is consistent with the experimentally measured emission spectrum [Fig. 1(d)] and with the laser near-field symmetry [Figs. 2(a) and 2(b)]. Figures 3(a) and 3(b) show the electric field component normal to the semiconductor-metal surface $\left(E_{z}\right)$ and its in-plane spatial Fourier transform $\left(\widetilde{E}_{z}\right)$ for the $B_{1}$ mode. It is interesting to note that this mode has only a small overlap with the central region of the PC cavity, a characteristic which may partially explain its preferential selection as the lasing mode, due to the expected in-plane spreading resistance in the etched PC region and consequent higher current injection and gain in the periphery of the PC. Calculations of the vertically emitted radiation field, taken in a plane several wavelengths above the cavity surface, were performed by eliminating the nonpropagating FDTD near-field components and introducing, to simulate the experimental conditions, a $30^{\circ}$ collection angle cut-off for the imaging optics. The resulting $\hat{x}$ - and $\hat{y}$-polarized intensity patterns of the $B_{1}$ mode are shown in Figs. 2(c) and 2(d), and closely match the polarized micro-bolometer camera images [Figs. 2(a) and 2(b)].

As a final consistency check, far-field emission measurements and calculations were performed. Figure 3(c) shows a typical far-field intensity pattern of the $B_{1}$ lasing mode, measured by scanning a $300 \mu \mathrm{m} \times 300 \mu \mathrm{m}$ nitrogen-cooled $\mathrm{HgCdTe}$ detector in a plane parallel to the semiconductor chip surface at a distance of approximately $10 \mathrm{~cm}$ without any intermediate optics. The theoretical far-field emission to AlP license or copyright, see http://apl.aip.org/apl/copyright.jsp 

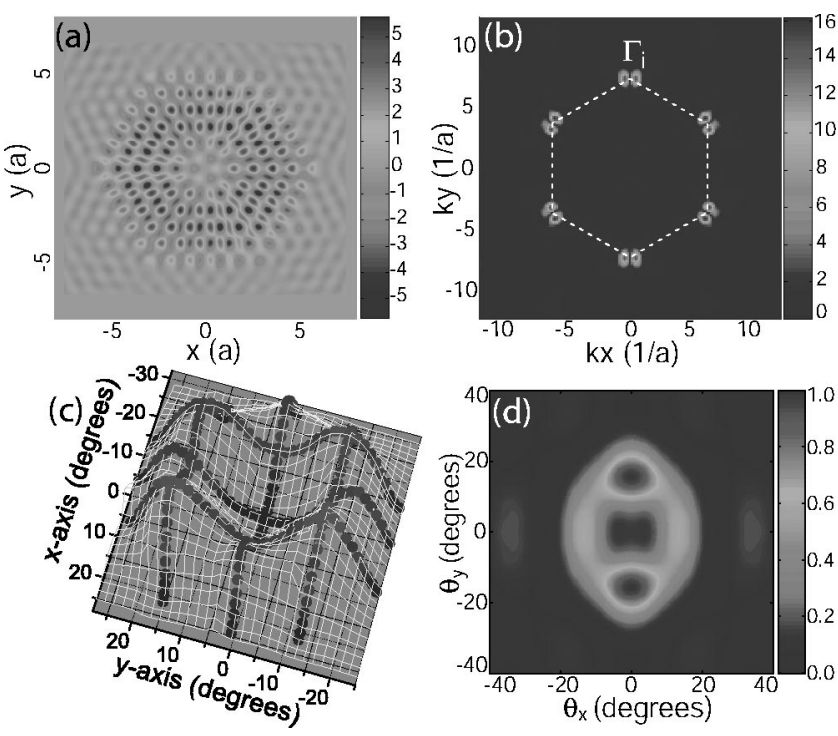

FIG. 3. FDTD-generated plot of (a) $E_{z}$ and (b) $\widetilde{E}_{z}$ for the $B_{1}$ mode in the plane of the PC cavity just beneath the metal contact in the semiconductor active region. (c) Far-field emission pattern of the PC microcavity laser. The experimental data are shown as dots, and an interpolation of the line scans (shown as a light grid) is used to generate an approximate 2D intensity image. (d) FDTD simulation of the far-field intensity pattern for the highfrequency mode within the $A$-peak set of resonances.

pattern was calculated by transforming the FDTD generated radiation field into the far-field ${ }^{13}$ and is shown in Fig. 3(d). The increased intensity of the two lobes on the $\hat{y}$ axis in Fig. 2(c) of the $\hat{x}$-polarized intensity and in Fig. 3(d) of the farfield pattern is likely a result of inadvertent symmetry breaking of the hexagonal symmetry of the PC cavity in the FDTD simulation. ${ }^{14}$ In this case, the computed near-field under the metal contact is still very symmetric, as shown in Fig. 3(a). The symmetry breaking in the measured far-field (experimentally we observe the opposite effect, more intensity in the $\hat{y}$ polarization), is most likely a result of the rectangular metal contact geometry and/or non-uniform current injection.

The FDTD-calculated radiative quality factor $(Q)$ of the $B_{1}$ mode was found to be roughly a factor of 2 higher than the $Q$ value of the neighboring $A$-peak resonant modes, helping explain why it is the first mode to lase. The calculated in-plane, top, and substrate radiation rates, as given by effective cavity quality-factors, were $Q_{\|}=600, Q_{t}=10000$, and $Q_{s}=7000$, respectively. An estimate for the $Q$ value associated with internal loss in the metal and semiconductor at 8 $\mu \mathrm{m}\left(\alpha_{\mathrm{i}}=40 \mathrm{~cm}^{-1}\right)$ is $Q_{a}=800$. Thus, the total vertical extraction efficiency of the PC microcavity laser is estimated to be $\eta_{t}=Q_{t}^{-1} /\left(Q_{\|}^{-1}+Q_{t}^{-1}+Q_{s}^{-1}+Q_{a}^{-1}\right)=3 \%$. The vertical extraction of light in this case is due to the radiation of small in-plane Fourier components near the $\Gamma$ point of the hexagonal PC reciprocal lattice [Fig. 3(b)], as in second-order Bragg diffraction. The coupling of radiation from the bottom semiconductor-metal interface to the top metal-air interface (from which the radiation finally escapes) is mediated through the air holes, ${ }^{11}$ as well as through the metal itself (although this last effect has not been included in our simulations).

In summary, we have used a combination of near- and far-field measurements to determine the symmetry of the lasing mode. The results, in terms of vertical emission pattern and lasing wavelength, are in good agreement with the 3DFDTD simulations. We believe that the reduction of the farfield emission pattern symmetry may be due to subtle nearfield asymmetries resulting from the rectangular top metal contact pad or nonuniform current injection.

This work was partly supported by DARPA/ARO under Contract No. DAAD19-00-C-0096 and by the Charles Lee Powell Foundation. The authors thank R. Martini for lending them the microbolometer camera. K.S. thanks the Hertz Foundation for its financial support.

${ }^{1}$ R. Colombelli, K. Srinivasan, M. Troccoli, O. Painter, C. Gmachl, D. M. Tennant, A. M. Sergent, D. L. Sivco, A. Y. Cho, and F. Capasso, Science 302, 1374 (2003).

${ }^{2}$ O. Painter, R. K. Lee, A. Yariv, A. Scherer, J. D. O'Brien, P. D. Dapkus, and I. Kim, Science 284, 1819 (1999).

${ }^{3}$ L. M. Miller, G. D. Smith, and G. L. Carr, J. Biol. Phys. 29, 219 (2003).

${ }^{4}$ K. E. Meissner, P. L. Gourley, T. M. Brennan, and B. E. Hammons, Appl. Phys. Lett. 69, 1517 (1996).

${ }^{5}$ B. D'Urso, O. Painter, J. O'Brien, T. Tombrello, A. Scherer, and A. Yariv, J. Opt. Soc. Am. B 15, 1155 (1998).

${ }^{6}$ K. Sakoda, Phys. Rev. A 52, 8992 (1995).

${ }^{7}$ M. Tinkham, Group Theory and Quantum Mechanics, International Series in Pure and Applied Physics (McGaw-Hill, New York, 1964).

${ }^{8}$ J. D. Joannopoulos, R. D. Meade, and J. N. Winn, Photonic Crystals (Princeton University Press, Princeton, NJ, 1995).

${ }^{9}$ O. Painter and K. Srinivasan, Phys. Rev. B 68, 035110 (2003).

${ }^{10}$ For a hexagonal cavity, one must consider the $C_{6 v}$ symmetry point group, which supports a non-degenerate $B_{1}$ IRREP that shares the same transformation properties of the $B_{1}$ IRREP of $C_{2 v}$ under the symmetries of a rectangle (the mode may also be from a degenerate $E_{1}$ IRREP space of $C_{6 v}$, although FDTD simulations show this is not the case).

${ }^{11}$ L. Martin-Morteno, F. J. Garcia-Vidal, H. J. Lezec, K. M. Pellerin, T. Thio, J. B. Pendry, and T. W. Ebbesen, Phys. Rev. Lett. 86, 1114 (2001).

${ }^{12}$ J. D. Jackson, Classical Electrodynamics, 2nd ed. (Wiley, New York, 1975).

${ }^{13}$ J. B. Judkins and R. W. Ziolkowski, J. Opt. Soc. Am. A 12, 1974 (1995).

${ }^{14}$ This may result from discretization error in employing the mirror boundary conditions and/or the rectangular boundary of the simulation volume. 